\title{
Neutron Scattering Study of the Field-Dependent Ground State and the Spin Dynamics in Spin-One-Half $\mathrm{NH}_{4} \mathrm{CuCl}_{3}$
}

\author{
Ch. Rüegg, ${ }^{1, *}$ M. Oettli, ${ }^{1}$ J. Schefer, ${ }^{1}$ O. Zaharko, ${ }^{1}$ A. Furrer, ${ }^{1}$ H. Tanaka, ${ }^{2}$ K. W. Krämer, ${ }^{3}$ H.-U. Güdel,,${ }^{3}$ P. Vorderwisch,${ }^{4}$ \\ K. Habicht, ${ }^{4,5}$ T. Polinski, ${ }^{4}$ and M. Meissner ${ }^{4}$ \\ ${ }^{1}$ Laboratory for Neutron Scattering, ETH Zurich and Paul Scherrer Institute, 5232 Villigen PSI, Switzerland \\ ${ }^{2}$ Department of Physics, Tokyo Institute of Technology, 152-8551 Tokyo, Japan \\ ${ }^{3}$ Department for Chemistry and Biochemistry, University of Berne, 3000 Bern 9, Switzerland \\ ${ }^{4}$ BENSC, Hahn-Meitner Institute, 14109 Berlin, Germany \\ ${ }^{5}$ Institute of Solid State Physics, Technical University of Darmstadt, 64289 Darmstadt, Germany
}

(Received 27 April 2004; published 16 July 2004)

\begin{abstract}
Elastic and inelastic neutron scattering experiments have been performed on the dimer spin system $\mathrm{NH}_{4} \mathrm{CuCl}_{3}$, which shows plateaus in the magnetization curve at $m=1 / 4$ and $m=3 / 4$ of the saturation value. Two structural phase transitions at $T_{1} \approx 156 \mathrm{~K}$ and at $T_{2}=70 \mathrm{~K}$ lead to a doubling of the crystallographic unit cell along the $b$ direction and as a consequence a segregation into different dimer subsystems. Long-range magnetic ordering is reported below $T_{N}=1.3 \mathrm{~K}$. The magnetic field dependence of the excitation spectrum identifies successive quantum phase transitions of the dimer subsystems as the driving mechanism for the unconventional magnetization process in agreement with a recent theoretical model.
\end{abstract}

DOI: 10.1103/PhysRevLett.93.037207

The current experimental and theoretical interest in the magnetic ground state and the excitation spectrum of model spin systems is related to the numerously observed phenomena directly originating from quantum effects. The successive appearance of plateaus at fractional values $0 \leq m \leq 1$ of the complete magnetic saturation $(m=1)$ in an external magnetic field is a recent example. They are observed in $\mathrm{Cu}^{2+}$-based $S=1 / 2$ compounds with different dimensionalities, such as the $2 \mathrm{D}$ orthogonal $\mathrm{SrCu}_{2}\left(\mathrm{BO}_{3}\right)_{2}$ [1] and the 3D $\mathrm{NH}_{4} \mathrm{CuCl}_{3}$ [2] dimer spin systems. The former shows distinct plateaus at $m=1 / 8$, $1 / 4$, and $1 / 3$, the latter at $m=1 / 4$ and $3 / 4$. Further evidence is reported for the frustrated quasi-2D spin system $\mathrm{Cs}_{2} \mathrm{CuBr}_{4}$ [3], as well as the integer spin $S=1$ 3D dimer [4] and $1 \mathrm{D} \mathrm{Ni}^{2+}$-based exchange-alternating chain compounds [5]. A necessary condition to observe a magnetization plateau in quantum spin systems has been formulated by Oshikawa et al. for one and arbitrary dimensions [6,7] and is based on an extended LiebSchultz-Mattis theorem. But the microscopic mechanisms leading to the explicit or spontaneous breaking of translational symmetry in the ground state are not specified, which motivates the detailed investigation of the different compounds.

In the present Letter, the results of neutron scattering experiments and measurements of the specific heat on $\mathrm{NH}_{4} \mathrm{CuCl}_{3}$ single crystals are presented. Two phase transitions have been observed leading to a low-temperature crystal structure with the space group $P \overline{1}$ and a doubling of the unit cell along the $b$ direction. As a direct consequence, the $\mathrm{Cu}^{2+}$ dimers segregate into different subsystems. Inelastic neutron scattering experiments (INS) in high magnetic fields further prove that the unconventional magnetization curve originates from the successive
PACS numbers: 75.10.Jm, 75.40.Cx, 75.40.Gb, 78.70.Nx

quantum phase transitions of these nearly decoupled dimer subsystems with individual spin energy gaps, similar to the transition observed in $\mathrm{TlCuCl}_{3}[8,9]$. We therefore conclude that the magnetization plateaus in $\mathrm{NH}_{4} \mathrm{CuCl}_{3}$ are rather a direct consequence of an explicit and not a spontaneous breaking of translation symmetry, in contrast to $\mathrm{SrCu}_{2}\left(\mathrm{BO}_{3}\right)_{2}$ but in agreement with a recently proposed theoretical model described in Ref. [10].

The space group of $\mathrm{NH}_{4} \mathrm{CuCl}_{3}$ has been reported to be $P 2_{1} / c$ at room temperature [11], isostructural to the related antiferromagnetic (AF) dimer systems $\mathrm{TlCuCl}_{3}$ and $\mathrm{KCuCl}_{3}$ with a singlet-triplet spin energy gap [12]. But the magnetization in the $\mathrm{Tl}$ and $\mathrm{K}$ compounds increases continuously as a function of field above the gap value $(m=0$ plateau) $[13,14]$. Elastic neutron scattering experiments have been performed on the single crystal diffractometers TOPSI and TriCS at the spallation neutron source SINQ, Paul Scherrer Institute, to investigate structural changes as a possible explanation for the obvious differences within the $\mathrm{ACuCl}_{3}$ family of compounds. A single crystal [dimensions $(3 \times 1 \times 6) \mathrm{mm}^{3}$, sample preparation described in Ref. [2]] has been mounted inside a closed cycle refrigerator or a ${ }^{4} \mathrm{He}$ flow cryostat on a Eulerian cradle, depending on the required temperature range. Using cold neutrons with $\lambda=4.74 \AA$ from a pyrolitic graphite (PG) monochromator and a $\mathrm{Be}$ filter in front of the sample position, measurements have been performed on the instrument TOPSI to focus on the $\left(\begin{array}{lll}0 & 0 & \ell\end{array}\right)$ direction. In Fig. 1(c), the temperature dependence of the integrated intensity measured at the $\left(\begin{array}{lll}0 & 0 & 1\end{array}\right)$ position relative to the strong $\left(\begin{array}{lll}0 & 0 & 2\end{array}\right)$ Bragg peak is presented. A considerable increase is reported below $T_{1} \approx 156 \mathrm{~K}$.

Rocking curves of the nuclear Bragg peaks in the $a^{*} c^{*}$ scattering plane have been measured on the instrument 

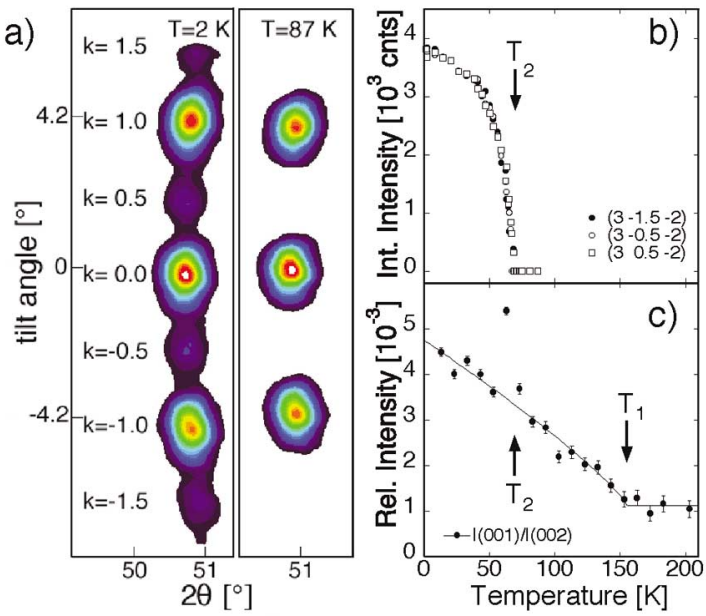

FIG. 1 (color). (a) $(3 k-2)$ Bragg peaks above and below the structural transition at $T_{2}=70 \mathrm{~K}$. The crystallographic unit cell is doubled along the $b$ direction. (b) Integrated intensities of $(3 k-2)$ Bragg peaks with half-integer $k$ as a function of temperature. (c) Temperature dependence of the relative intensities of ( $\left(\begin{array}{llll}0 & 0 & 1\end{array}\right)$ and $\left(\begin{array}{lll}0 & 0 & 2\end{array}\right)$ nuclear Bragg peaks with an increase below $T_{1} \approx 156 \mathrm{~K}$. The solid line is a guide to the eye.

TriCS between room temperature and $T=2 \mathrm{~K}$ using neutrons with $\lambda=1.176 \AA$ from a Ge monochromator. A projection of a typical $\omega$ scan onto the two-dimensional detector, which allows simultaneous data collection of the $(h k \ell$ ) reflections with $-2<k<2$, is presented in Fig. 1(a). The striking observation is the appearance of Bragg peaks with half-integer Miller indices $k$ at low temperatures. Their temperature dependence is presented in Fig. 1(b). The integrated intensity shows an increase at $T_{2}=70 \mathrm{~K}$. No half-integer peaks could be detected along the $a$ and $c$ axes.

In contrast to $\mathrm{TlCuCl}_{3}$ and $\mathrm{KCuCl}_{3}$, the rotational dynamics of the $\mathrm{NH}_{4}^{+}$complexes in $\mathrm{NH}_{4} \mathrm{CuCl}_{3}$ introduces a new lattice degree of freedom, whose freezing at low temperatures might be the origin of the observed transitions. We emphasize that the ${ }^{2} \mathrm{H}$ - and ${ }^{1} \mathrm{H}$-resonance lines measured with NMR on deuterated and protonated powder samples are split at $T \approx 150 \mathrm{~K}$ [15]. Further evidence for a structural transition in $\mathrm{NH}_{4} \mathrm{CuCl}_{3}$ is reported in an early IR absorption study [16] as well as in ultrasonic experiments with anomalies in the temperature dependence of the elastic constants occurring at $T \approx 70 \mathrm{~K}$ [17]. Recent NMR results additionally show a splitting of the ${ }^{15} \mathrm{~N}$-resonance line around this temperature [18].

From the refinement of our neutron diffraction data, we confirm that $\mathrm{NH}_{4} \mathrm{CuCl}_{3}$ crystallizes in the monoclinic space group $P 2_{1} / c$ at room temperature $[a=4.012(6) \AA$, $b=14.18(4) \AA, c=8.92(1) \AA, \beta=96.25(8)^{\circ}, 138$ reflections, $R=2.18 \%]$. However, at $T_{1}$ the appearance of the $\left(\begin{array}{lll}0 & 0 & 1\end{array}\right)$ reflection implies symmetry lowering, namely, vanishing of the $c$ glide plane. The possible space groups for $T_{2}<T<T_{1}$ are therefore $P 2_{1}$ and $P \overline{1}$ with the same dimension of the unit cell. Below $T_{2}(h k \ell)$ reflections are observed with half-integer Miller indices $k$, corresponding to a doubling of the unit cell along $b$. The lowtemperature phase, sketched in Fig. 2, can therefore be only of $P \overline{1}$ or $P 1$ symmetry, even if a complete refinement cannot be provided on the basis of our limited data set $[a=3.943(2) \AA, b=28.19(4) \AA, c=8.913(3) \AA, \alpha=$ $90.21(7)^{\circ}, \beta=95.42(3)^{\circ}, \gamma=89.79(7)^{\circ}$, at $\left.T=2 \mathrm{~K}\right]$. Supposing the preservation of a center of inversion at low temperatures, which is motivated beneath, at $T_{1}$ a transition $P 2_{1} / c \rightarrow P \overline{1}$ is proposed, with two different types of dimers, followed at $T_{2}$ by $P \overline{1} \rightarrow P \overline{1}$ with a doubling along $b$. The resulting inequivalent $\mathrm{Cu}^{2+}$ dimer sites, in the following labeled $\mathrm{A}, \mathrm{B}$, and $\mathrm{C}$, have volume fractions of $25 \%, 50 \%$, and $25 \%$, respectively. Simultaneously four different $\mathrm{NH}_{4}^{+}$groups are distinguished in agreement with the low-temperature ${ }^{15} \mathrm{~N}-\mathrm{NMR}$ results, where the single resonance line progressively splits into four lines below $T_{2}$. This splitting has been interpreted in terms of a dimer superstructure along the $a$ axis [18], which is not supported by our neutron diffraction data. We can exclude that the lowtemperature structure of $\mathrm{NH}_{4} \mathrm{CuCl}_{3}$ is characterized by the space group $P 1$, since in that case the subsystem $\mathrm{B}$ would additionally decompose into two subsystems B and $\mathrm{B}^{\prime}$ (see Fig. 2), with volume fractions of $25 \%$ each, and eight different $\mathrm{NH}_{4}^{+}$groups would be expected, which contradicts our INS results as well as the number of ${ }^{15} \mathrm{~N}-\mathrm{NMR}$ lines. This strongly suggests that the center of inversion is preserved, i.e., $\mathrm{NH}_{4} \mathrm{CuCl}_{3}$ has a $P \overline{1}$ crystal structure at $T=2 \mathrm{~K}$ with three inequivalent dimer sites.

The nature of the ground state has been investigated on the triple-axis spectrometer TASP at the spallation neutron source SINQ. Six $\mathrm{NH}_{4} \mathrm{CuCl}_{3}$ single crystals have been coaligned (total mass $0.5 \mathrm{~g}$, sample mosaic $1^{\circ}$ ) and mounted on a ${ }^{3} \mathrm{He}-{ }^{4} \mathrm{He}$ dilution insert inside a $9 \mathrm{~T}$ vertical cryomagnet. The orientation of the field is defined perpendicular to the plane spanned by the $b^{*}$ and $c^{*}$ reciprocal directions. The instrument has been operated using neutrons with $E_{i}=4.7 \mathrm{meV}$ from a focusing PG monochromator and a cold $\mathrm{Be}$ filter between the sample

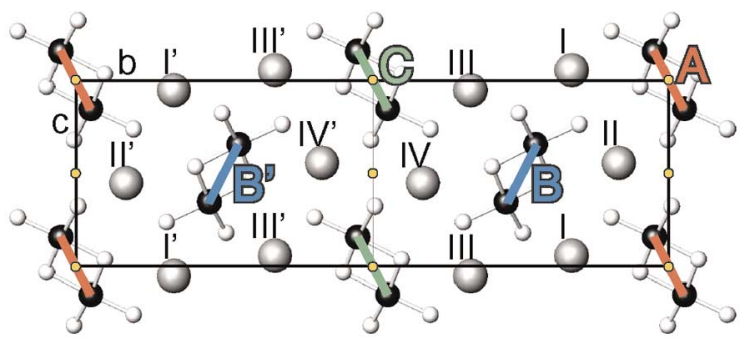

FIG. 2 (color). Low-temperature structure of $\mathrm{NH}_{4} \mathrm{CuCl}_{3}$ in projection along $a$ on the $b c$ plane. The high-temperature unit cell is doubled along the $b$ axis. Subsystems of $\mathrm{Cu}^{2+}$ dimers are marked by $\mathrm{A}, \mathrm{B}, \mathrm{B}^{\prime}$, and $\mathrm{C}$, inequivalent $\mathrm{NH}_{4}^{+}$groups by Roman numbers $\mathrm{I}-\mathrm{IV}$ and $\mathrm{I}^{\prime}-\mathrm{IV}^{\prime}$. Orange circles denote inversion centers in case of space group $P \overline{1}$, where the primed sites become equivalent to the unprimed ones. 
position and a flat PG analyzer. A characteristic increase of a magnetic Bragg peak is observed at the $Q=\left(\begin{array}{lll}0 & 0 & 1\end{array}\right)$ position on top of the discussed tiny structural contribution (see Fig. 3 inset). The temperature dependence of the peak intensity near the transition is fitted by the exponent $\beta=0.33(1)$ and $T_{N}=1.28(1) \mathrm{K}$ in the standard expression $I \propto\left(T_{N}-T\right)^{2 \beta}$. The peak intensity further shows a strong field dependence with a smooth maximum at $H \approx$ $3 \mathrm{~T}$ followed by a sharp decrease around $H=5 \mathrm{~T}$, where the onset of the $m=1 / 4$ plateau is observed. The lowfield part of the phase diagram has additionally been mapped by the field dependence of the low-temperature specific heat as summarized in Fig. 3.

From the absence of an $m=0$ plateau and the finite slope of the magnetization near zero field, a magnetically ordered ground state is expected in $\mathrm{NH}_{4} \mathrm{CuCl}_{3}$ [2]. The magnetic specific heat additionally shows a weak peak in zero field at $T=1.3 \mathrm{~K}$, as also reported in Ref. [19], and a characteristic splitting of the ${ }^{15} \mathrm{~N}-\mathrm{NMR}$ lines has been found in magnetic fields below the $m=1 / 4$ plateau at $T \approx 1.7 \mathrm{~K}$ [18]. The $Q=\left(\begin{array}{lll}0 & 0 & 1\end{array}\right)$ position, where the magnetic peak appears, corresponds to the AF zone center in $\mathrm{TlCuCl}_{3}$ and $\mathrm{KCuCl}_{3}$, which is unchanged by the structural transitions because the $c$ direction remains unaffected. The field dependence of the Bragg peak intensity exhibits the typical behavior for an ordered AF, where the spins are successively polarized along the field direction. We therefore conclude that $\mathrm{NH}_{4} \mathrm{CuCl}_{3}$ shows long-range AF ordering below $T_{N}=1.3 \mathrm{~K}$ in zero field. The observed $T_{N}(H)$ shows the characteristics of the field dependence of the transverse magnetic moment as calculated by Matsumoto [10] and confirms a suggested shape of the phase boundary [19]. A progressive ordering of $25 \%, 50 \%$, and $25 \%$ of the total spins is in agreement with the values of the magnetization at the first and at the

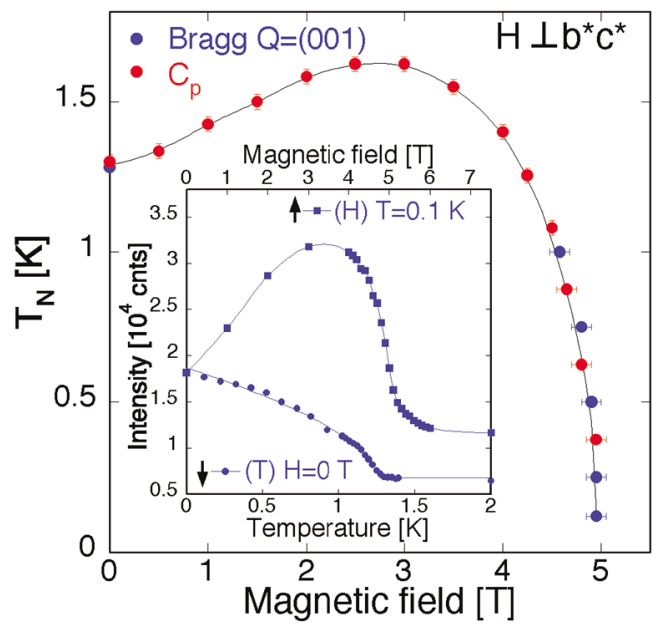

FIG. 3 (color). Low-field and low-temperature phase diagram of $\mathrm{NH}_{4} \mathrm{CuCl}_{3}$ from elastic neutron scattering and specific heat measurements. Inset: Temperature $(T), H=0 \mathrm{~T}$, and field $(H)$, $T=0.1 \mathrm{~K}$, dependence of the $\left(\begin{array}{lll}0 & 0 & 1\end{array}\right)$ AF Bragg peak. The solid lines are guides to the eye. second plateau as well as with the analysis of the magnetic entropy $[2,19]$. Thus, one of the subsystems with a fraction of $25 \%$, let us assume $\mathrm{A}$, orders below $T_{N}$, whereas $\mathrm{B}$ and $\mathrm{C}$ remain mainly in a singlet state.

Using the same multicrystal sample, INS experiments have been performed on the TASP instrument below $T_{N}$, and above $T_{N}$ on the triple-axis spectrometer V2 at the Berlin Neutron Scattering Center, Hahn-MeitnerInstitute, equipped with a $15 \mathrm{~T}$ vertical cryomagnet. Energy scans have been measured at the AF zone center $Q=\left(\begin{array}{lll}0 & 0 & 1\end{array}\right)$ with the same instrumental setup as described above, but using a focusing PG analyzer. The results are presented in Fig. 4. At $T=1.8 \mathrm{~K}>T_{N}$ two excitations, denoted in the following by $\mathrm{B}$ and $\mathrm{C}$, are observed in zero field at $E_{\mathrm{B}} \approx 1.6 \mathrm{meV}$ and $E_{\mathrm{C}} \approx$ $3.0 \mathrm{meV}$, which show linear Zeeman splittings as a function of the applied magnetic field. At temperatures below $T_{N}$, the excitation energies do not change within experimental error. A linear fit to the data collected at $T=$ $1.8 \mathrm{~K}$ yields $E_{\mathrm{B}}=1.593(5) \mathrm{meV}, g_{\mathrm{B}}=1.95(4)$ for the lower mode and $E_{\mathrm{C}}=2.987(9) \mathrm{meV}, g_{\mathrm{C}}=2.04(2)$ for the higher mode. The corresponding critical fields for the closing of the spin energy gaps are $H_{\mathrm{B}}=14$.1(4) $\mathrm{T}$ and $H_{\mathrm{C}}=25.3(4)$ T. An additional mode linearly increasing with field is observed for $H>H_{\mathrm{A}}$, with $H_{\mathrm{A}}=$ 4.9(2) $\mathrm{T}$ and $g_{\mathrm{A}}=2.05(3)$.

At $H=0 \mathrm{~T}$, the excitation energies $E_{\mathrm{B}}$ and $E_{\mathrm{C}}$ are in agreement with the results of the first inelastic neutron scattering experiments performed on $\mathrm{NH}_{4} \mathrm{CuCl}_{3}$ single crystals in zero field [20]. Two gapped excitations are reported at $E \approx 1.6$ and $3.0 \mathrm{meV}$, which coincide with the extrapolated zero-field frequencies of the ESR modes [21] and hardly show any dispersion [20]. The linear Zeeman splitting observed in our INS spectra directly proves that these two excitations have singlet-triplet nature. We relate the excitation energies to the individual

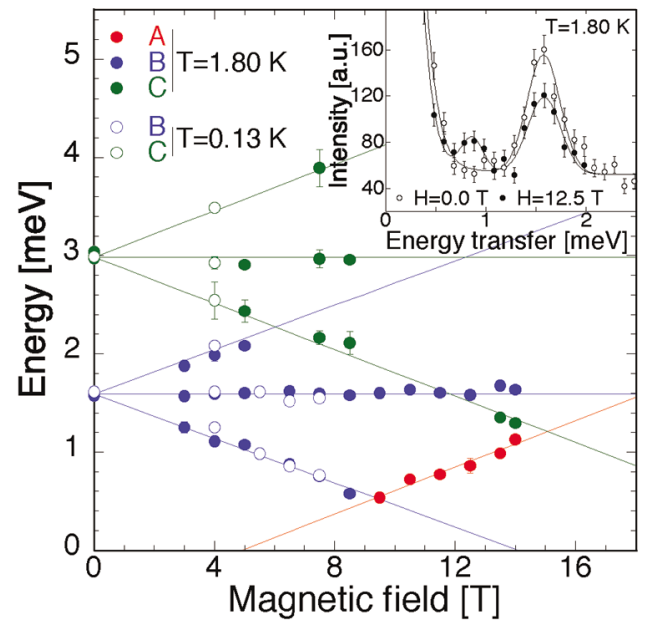

FIG. 4 (color). Field dependence of the spin dynamics in $\mathrm{NH}_{4} \mathrm{CuCl}_{3}$ measured at $Q=\left(\begin{array}{lll}0 & 01\end{array}\right), T=1.8 \mathrm{~K}$, and $T=$ $0.13 \mathrm{~K}$. Solid lines represent linear fits described in the text. Inset: INS spectra measured at $H=12.5 \mathrm{~T}$ and in zero field. 
spin gaps of the $\mathrm{B}$ and $\mathrm{C}$ dimer subsystems, which is additionally supported by the relation of their spectral weights. Strong incoherent scattering in combination with the intrinsically low inelastic signal limits our INS study to $E \geq 0.5 \mathrm{meV}$, but the linear field dependence is confirmed down to $0.2 \mathrm{meV}$ by ESR [17]. The critical fields of the magnetization plateaus at $T=0.5 \mathrm{~K}$ and for $H \| a$ are $H_{c 1}=5.0 \mathrm{~T}, H_{c 2}=12.8 \mathrm{~T}, H_{c 3}=17.9 \mathrm{~T}, H_{c 4}=$ $24.7 \mathrm{~T}$, and $H_{s}=29.1 \mathrm{~T}$ [2]. Accepting small quantitative deviations attributed to the different field orientation and temperature, the assignments $H_{\mathrm{B}}=H_{c 2}$ and $H_{\mathrm{C}}=$ $H_{c 4}$ are justified. The general increase of $H_{c 2}$ and $H_{c 4}$ at finite temperatures is further in agreement with the magnetization measurements [19]. We therefore conclude that in $\mathrm{NH}_{4} \mathrm{CuCl}_{3}$ the first plateau sets in, when the subsystem A is completely polarized along the field direction [10]. Accordingly, the second plateau starts, when also the subsystem B reaches its complete saturation. The magnetization rises at the edge of the first (second) plateau, when the individual spin gaps of the dimer subsystem B (C) are closed and field-induced magnetic ordering occurs, similar to the soft-mode transition reported for $\mathrm{TlCuCl}_{3}$ [9]. The energy splittings observed at $T=$ $0.13 \mathrm{~K}$ and $T=1.8 \mathrm{~K}$ are identical, i.e., they are not affected by the onset of magnetic ordering. This directly proves that $75 \%$ (B and C) of the spin system remain in a gapped singlet state whereas $25 \%$ (A) order. We further interpret the mode linearly increasing above $H_{\mathrm{A}}=H_{c 1}$ as the field-induced opening of a spin gap for the subsystem A. This gap can be understood as the linear increase of the Zeeman energy for a triplet-singlet transition.

Comprehensive experimental and theoretical studies of the magnetization plateaus in $\mathrm{SrCu}_{2}\left(\mathrm{BO}_{3}\right)_{2}$ have revealed that the spontaneous crystallization of triplet states in an enlarged rhomboid cell on the 2D plane of orthogonal AF dimers and a significant spin-lattice coupling explain the complex spin density profile observed by NMR [22-24]. It is important to notice that no structural transition leading to inequivalent dimer sites could be detected below the onset of the first plateau and especially in zero field [22,25]. The introduction of DzyaloshinskyMoriya interactions and of the aforementioned spinlattice effects is, however, essential to describe the details of the complex magnetization curve. In contrast, two structural transitions have been observed in the present experiments on $\mathrm{NH}_{4} \mathrm{CuCl}_{3}$. As a direct consequence, the $\mathrm{Cu}^{2+}$ dimers segregate into three, nearly decoupled, subsystems $\mathrm{A}, \mathrm{B}$, and $\mathrm{C}$ with volume fractions $25 \%, 50 \%$, and $25 \%$, respectively, according to the model proposed by Matsumoto [10], but with a different dimer superstructure. Because the $\mathrm{Cu}-\mathrm{Cl}-\mathrm{Cu}$ bond angle is close to the critical $90^{\circ}$, the related small structural distortions may be sufficient to cause the considerable differences in the $\mathrm{AF}$ intradimer exchange interactions estimated to be $J_{\mathrm{A}} \approx 0.3 \mathrm{meV}, J_{\mathrm{B}} \approx 1.8 \mathrm{meV}$, and $J_{\mathrm{C}} \approx 3.0 \mathrm{meV}$ [10].
To illustrate the importance of structural effects, we mention that the corresponding AF intradimer coupling constants $J$ differ by as much as $1.14 \mathrm{meV}$ in the related $\mathrm{Tl}$ and $\mathrm{K}$ compounds [12]. We therefore conclude that in contrast to $\mathrm{SrCu}_{2}\left(\mathrm{BO}_{3}\right)_{2}$ an explicit breaking of the translational symmetry is responsible for the magnetization plateaus observed in $\mathrm{NH}_{4} \mathrm{CuCl}_{3}$. This microscopic mechanism has to be considered as another possibility to explain the magnetization plateaus observed in other quantum spin systems.

We are indebted to M. Matsumoto, B. Normand, T. M. Rice, and M. Sigrist for enlightening discussions. We further thank B. Meier, M. Tomaselli, and J. Stahn for their expert support during part of the experiments. The work at SINQ, Paul Scherrer Institute, was financially supported by the Swiss National Science Foundation and the NCCR MaNEP project, and at BENSC, HahnMeitner Institute, by the EU through the FP6-NMI3 Access Activity.

*Electronic address: christian.rueegg@psi.ch

[1] H. Kageyama et al., Phys. Rev. Lett. 82, 3168 (1999).

[2] W. Shiramura et al., J. Phys. Soc. Jpn. 67, 1548 (1998).

[3] T. Ono et al., Phys. Rev. B 67, 104431 (2003).

[4] M. Uchida et al., Phys. Rev. B 66, 054429 (2002).

[5] Y. Narumi et al., Physica (Amsterdam) 246B-247B, 509 (1998).

[6] M. Oshikawa, M. Yamanaka, and I. Affleck, Phys. Rev. Lett. 78, 1984 (1997).

[7] M. Oshikawa, Phys. Rev. Lett. 84, 1535 (2000).

[8] M. Matsumoto, B. Normand, T. M. Rice, and M. Sigrist, Phys. Rev. Lett. 89, 077203 (2002).

[9] Ch. Rüegg et al., Nature (London) 423, 62 (2003).

[10] M. Matsumoto, Phys. Rev. B 68, 180403 (2003).

[11] R. D. Willett, C. Dwiggins, R. F. Kruh, and R. E. Rundle, J. Chem. Phys. 38, 2429 (1963).

[12] N. Cavadini et al., Phys. Rev. B 63, 172414 (2001).

[13] H. Tanaka et al., Physica (Amsterdam) 237B-238B, 120 (1997).

[14] A. Oosawa et al., Phys. Rev. B 66, 104405 (2002).

[15] Ch. Rüegg et al. (unpublished).

[16] A. M. Heyns and C. J. H. Schutte, J. Mol. Struct. 8, 339 (1971).

[17] S. Schmidt et al., Europhys. Lett. 53, 591 (2001).

[18] Y. Shimaoka et al., Physica (Amsterdam) 329B-333B, 894 (2003).

[19] B. Kurniawan et al., J. Phys. Condens. Matter 11, 9073 (1999).

[20] A. Oosawa, T. Ono, K. Kakurai, and H. Tanaka, condmat/0304172.

[21] B. Kurniawan et al., Phys. Rev. Lett. 82, 1281 (1999).

[22] K. Kodama et al., Science 298, 395 (2002).

[23] S. Miyahara, F. Becca, and F. Mila, Phys. Rev. B 68, 024401 (2003).

[24] T. M. Rice, Science 298, 760 (2002).

[25] B. Wolf et al., Phys. Rev. Lett. 86, 4847 (2001). 